Commun. Fac. Sci. Univ. Ank. Series $A_{1}$ V. 37, pp 5-8 (1988)

\title{
THE 3- PLANE AND THE LIGHT CONE
}

\section{E.M. ELKHOLY and S.M. AL-AREEFI}

\section{ABSTRACT}

In this paper we show that the 3-plane passing through the origin in a space-time will intersect the light cone in two perpendicular 2-planes.

\section{1- The Principal Planes:}

In this section we will give a sketch of how the principal planes can be obtained in order to be able to discuss the way in which a 3-plane intersect the light cone in space time.

A principal plane is a diametral plane which is at right angles to the chords which it bisects. Now if the axes are rectangular, the diametral plane (whose equation is

$$
\begin{aligned}
& \imath \frac{\partial \mathbf{F}}{\partial \mathbf{x}}+\mathbf{m} \frac{\partial \mathbf{F}}{\partial \mathbf{y}}+\mathbf{n} \frac{\partial \mathbf{F}}{\partial \mathbf{z}}=\mathbf{0}, \text { where } \\
& \mathbf{F}(\mathrm{x}, \mathrm{y}, \mathrm{z}) \equiv \mathrm{ax}^{2}+\mathrm{by}^{2}+\mathbf{c z}^{2}+2 \mathrm{fyz}+2 \mathrm{gzx}+2 \mathrm{hxy}+\mathrm{d}=0 \\
& \text { or } \mathrm{x}(\mathbf{a} \iota+\mathbf{h m}+\mathbf{g n})+\mathrm{y}(\mathbf{h} \iota+\mathbf{b m}+\mathbf{f n})+\mathrm{z}(\mathbf{g} \boldsymbol{}+\mathbf{f m}+\mathbf{c n})=0)
\end{aligned}
$$

is at right angles to the line $\frac{\mathrm{x}}{\mathrm{l}}=\frac{\mathrm{y}}{\mathrm{m}}=\frac{\mathrm{z}}{\mathrm{n}}$, if

$$
\frac{\mathbf{a} \iota+\mathbf{h m}+\mathbf{g n}}{\iota}=\frac{\mathbf{h} \iota+\mathbf{b m}+\mathbf{f n}}{\mathbf{m}}=\frac{\mathbf{g} \iota+\mathbf{f m}+\mathbf{c n}}{\mathbf{n}}
$$

If each of these ratios is equal to $\lambda$, then

$$
\begin{aligned}
& (\mathbf{a}-\lambda) \mathfrak{b}+\mathbf{h m}+\mathbf{g n}=\mathbf{0}, \\
& \quad \mathbf{h}:+(\mathbf{b}-\lambda) \mathbf{m}+\mathbf{f} \mathbf{n}=\mathbf{0}, \\
& \quad \mathrm{g} \iota+\mathbf{f m}+(\mathrm{c}-\lambda) \mathbf{n}=\mathbf{0}
\end{aligned}
$$


Therefore, $\lambda$ is a root of the equation:

$$
\left|\begin{array}{ccc}
\mathbf{a}-\lambda & \mathbf{h} & \mathbf{g} \\
\mathbf{h} & b-\lambda & \mathbf{f} \\
\mathrm{g} & \mathbf{f} & \mathbf{c}-\lambda
\end{array}\right|=0
$$

$0_{i}$ equivalently,

$$
\lambda^{3}-\lambda^{2}(a+b+c)+\lambda\left(b c+c a+a b-h^{2}-g^{2}-\mathbf{f}^{2}\right)-\mathbf{D}=0
$$

where

$$
D \equiv\left|\begin{array}{lll}
a & h & g \\
\mathbf{b} & b & f \\
g & f & c
\end{array}\right|
$$

Equation (ii) is called the discriminating cubic. It gives three values of $\lambda$, to each of which corresponds a set of values for $(l, m, n)$ and by substituting these sets in the equation $b \frac{\partial \mathbf{F}}{\partial \mathbf{x}}+\mathbf{m} \frac{\partial \mathbf{F}}{\partial \mathbf{y}}+\mathbf{n} \frac{\partial \mathbf{F}}{\partial \mathbf{z}}=0$, which by means of the relations (i) reduces to $\lambda(c x+m y+n z)=0$, we obtain the equations of three principal planes $[1]$.

2- The Main Result:

A 3-plane in space time has the equation $\Sigma A_{\mathbf{r}} \mathbf{x}_{\mathrm{r}}+\mathrm{B}=\mathbf{0}, \mathbf{r}=$ $1,2,3,4$. This equation will reduce to

$$
\sum \mathrm{A}_{\mathrm{r}} \mathbf{x}_{\mathrm{r}}=0, \mathbf{r}=1,2,3,4,
$$

if the 3 -plane is passing through the origin. In this case it will have a unique orthogonal line through the origin with equations $\mathbf{x}_{\mathrm{r}}=\mathbf{A}_{\mathbf{r}} \mathbf{u}$, where $u$ is a parameter.

Now consider the equation of the light cone:

$$
\sum_{i=1}^{3} x^{2}-x_{4}^{2}=0=\langle x, x>=0
$$

and rewrite equation (1) as follows:

$$
\sum_{\mathbf{i}=1}^{3} \mathbf{B}_{\mathrm{i}} \mathbf{x}_{\mathbf{i}}+\mathbf{x}_{4}=0, \mathbf{B}_{\mathbf{i}}=\mathbf{A}_{\mathbf{i}} / \mathbf{A}_{4}
$$

From (1)' and (2) we have

$$
\left(\sum_{i=1}^{3} B_{i} x_{i}\right)^{2}=\left(-x_{4}\right)^{2}=x_{4}^{2}=\sum_{i=1}^{\sum} x^{2}
$$




$$
\therefore \sum_{i=1}^{3} B^{2}{ }_{i} x^{2}{ }_{i}+2 \sum_{\substack{i, j=1 \\ i \neq j}}^{3} B_{i} B_{j} x_{i} x_{j}=\sum_{i=1}^{3} x^{2}
$$

or

$$
\sum_{i=1}^{3} C_{i}^{2} x^{2}{ }_{i}+2 \sum_{\substack{i, j=1 \\ \mathbf{i} \neq j}}^{3} B_{i} B_{j} x_{i} x_{j}=0, C^{2}=B_{i}^{2}-1
$$

The discriminating cubic for equation (3) is:

$$
\begin{aligned}
& \lambda^{3}-\lambda^{2}\left(\mathrm{C}_{2}^{2}+\mathrm{C}_{2}^{2}+\mathrm{C}_{3}^{2}\right)+\lambda\left(\mathrm{C}_{2}{ }_{2} \mathrm{C}_{3}{ }_{3}+\mathrm{C}_{2}{ }_{1} \mathrm{C}_{3}^{2}+\mathrm{C}_{1}^{2} \mathrm{C}_{2}^{2}-\right. \\
& \left.-\mathbf{B}_{2}^{2} \mathbf{B}_{3}^{2}-\mathbf{B}_{1}^{2} \mathrm{~B}^{2}{ }_{3}-\mathbf{B}_{1}^{2} \mathbf{B}_{2}^{2}\right)-\mathbf{D}=0,
\end{aligned}
$$

where

$$
\mathbf{D} \equiv\left|\begin{array}{lll}
\mathrm{C}_{1}^{2} & \mathrm{~B}_{1} \mathrm{~B}_{2} & \mathrm{~B}_{1} \mathrm{~B}_{3} \\
\mathrm{~B}_{2} \mathrm{~B}_{1} & \mathrm{C}_{2}^{2} & \mathrm{~B}_{2} \mathrm{~B}_{3} \\
\mathrm{~B}_{1} \mathrm{~B}_{3} & \mathrm{~B}_{2} \mathrm{~B}_{3} & \mathrm{C}_{3}^{2}
\end{array}\right|=\mathrm{C}^{2}{ }_{1}+\mathrm{C}_{2}^{2}+\mathrm{C}_{3}^{2}+2 \neq 0
$$

Thus: $\lambda^{3}-\mathrm{A} \lambda^{2}-(2 \mathrm{~A}+3) \lambda-(\mathrm{A}+2)=0, \mathrm{~A} \stackrel{\mathrm{an}_{2}}{=} \mathrm{C}_{1}^{2}+\mathrm{C}_{2}^{2}$ $+\mathrm{C}_{3}^{2}$, and so, $(\lambda+1)^{2}[\lambda-(\mathrm{A}+2)]=0$. It follows that $\lambda=-1,-1$, $\mathrm{A}+2$.

Consider first $\lambda=-1$. In this case the set of equations (i) may reduce to the single equation:

$$
\mathrm{B}_{1} \iota_{1}+\mathrm{B}_{2} \mathrm{~m}_{1}+\mathrm{B}_{3} \mathrm{n}_{1}=0, \mathrm{i}=1,2,3,
$$

If we consider $\lambda=A+2$, the set of equations ( $\mathrm{i}$ ) may take the from:

$$
\begin{aligned}
& \left(\mathrm{B}_{2}^{2}+\mathrm{B}_{3}^{2}\right)_{3}+\mathbf{B}_{1} \mathbf{B}_{2} \mathbf{m}_{3}+\mathbf{B}_{1} \mathbf{B}_{3} \mathbf{n}_{3}=0 \\
& \mathbf{B}_{1} \mathbf{B}_{2 b_{3}}-\left(\mathbf{B}^{2}{ }_{1}+\mathbf{B}_{2}^{2}\right) \mathbf{m}_{3}+\mathbf{B}_{2} \mathbf{B}_{3} \mathbf{n}_{3}=0 \text {, } \\
& B_{1} B_{3} l_{3}+B_{2} B_{3} m_{3}-\left(B_{1}^{2}+B_{2}^{2}\right) n_{3}=0
\end{aligned}
$$

Dividing the first equation of (5) by $B_{1}$ and the second by $B_{2}$ then substracting, we have:

$\frac{\iota_{3}}{\mathbf{B}_{1}}=\frac{\mathbf{m}_{3}}{\mathbf{B}_{2}}$

Again from the second and third equations of (5), we get

$$
\frac{\mathbf{m}_{3}}{\mathbf{B}_{2}}=\frac{\mathbf{n}_{3}}{\mathbf{B}_{3}}
$$

Thus, 


$$
\frac{\iota_{3}}{\overline{\mathbf{B}}_{1}}=\frac{\mathbf{m}_{3}}{\mathbf{B}_{2}}+\frac{\mathbf{n}_{3}}{\mathbf{B}_{3}}
$$

From the above results we find that the single equation (4) corresponding to $\lambda_{1}=\lambda_{2}=-1$, is the condition that the directions given by $\left(\iota_{1}, m_{1}, n_{1}\right)$ and $\left(\iota_{3}, m_{3}, n_{3}\right)$ should be at right angles. The principal planes corresponding to the dircetions $\left(\iota_{1}, m_{1}, n_{1}\right)$ and $\left(\iota_{3}, m_{3}, n_{3}\right)$ are respectively: $\iota_{1} \mathbf{x}_{1}+\mathbf{m}_{1} \mathbf{x}_{2}+\mathbf{n}_{1} \mathbf{x}_{3}=0$ and $\iota_{3} \mathbf{x}_{1}+\mathbf{m}_{3} \mathbf{x}_{2}+\mathbf{n}_{3} \mathbf{x}_{3}=0$, or equivalently: $\iota_{1} \mathbf{x}_{1}+\mathbf{m}_{1} \mathbf{x}_{2}+\mathbf{n}_{1} \mathbf{x}_{3}=0$ and $B_{1} \mathbf{x}_{1}+B_{2} \mathbf{x}_{2}+B_{3} \mathbf{x}_{3}=0$, where $B_{1 l_{1}}+B_{2} m_{1}+B_{3} n_{1}=0$. It follows that the 3-plane which pass through the origin will intersect the light cone in two perpendicular planes.

\section{REFERENCE}

1- T.J. WII.LMORF, An Introduction to Differential Geometry, Oxford Unicersity Press, London, 2nd. Impression, 1982.

Dept. of Mathematics,

Faculty of Scioner

Tanta University,

Tanta, Egypt.
Department of Mathematics,

College of Science for Girls,

Dammam, Saudi Arabia. 